Políticas de vivienda

\title{
Pragmatismo, desaliento de expectativas y gobernabilidad. La política de villas de las gestiones de Mauricio Macri en la Ciudad Autónoma de Buenos Aires (2007-2015)
}

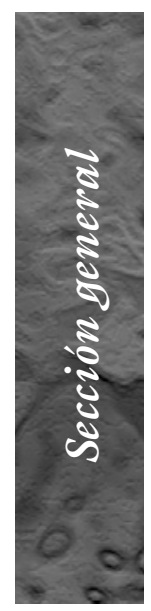

Pragmatism, Discouragement of Expectations and Governability. The Public Policies Towards Slums of Mauricio Macri Administration in the City of Buenos Aires (2007-2015)

Pragmatismo, desalento de expectativas e governabilidade. A politica de vilas das gestões de Mauricio Macri na Cidade Autónoma de Buenos Aires (2007-2015)

Carla Fainstein *

Ariel Matías Palombi**

Recibido: 31 de octubre de 2017

Aprobado: 24 de abril de 2018

Doi: http://dx.doi.org/10.12804/revistas.urosario.edu.co/territorios/a.6208

Para citar este artículo:

Fainstein, C., \& Palombi, A. M. (2019). Pragmatismo, desaliento de expectativas y gobernabilidad. La política de villas de las gestiones de Mauricio Macri en la Ciudad Autónoma de Buenos Aires (2007-2015). Territorios (40), 227-244. Doi: http://dx.doi.org/10.12804/revistas.urosario.edu.co/territorios/a.6208

\begin{abstract}
* Magister en Estudios Urbanos, Universidad Nacional de General Sarmiento. Becaria doctoral CONICET con sede en Instituto del Conurbano-UNGS. Área de estudio: Estudios urbanos. Correo electrónico: carla. fainstein@gmail.com. ORCID: https://orcid. org/0000-0002-6504-0586

** Licenciado en Sociología, Universidad de Buenos Aires INFOHABITAT - UNGS. Área de estudio: Estudios Urbanos. Correo electrónico: arielpalombi@ hotmail.com. ORCID: https://orcid.org/00000002-4155-5876
\end{abstract}


Palabras clave

Politicas públicas, asentamientos informales, organizaciones sociales, judicialización, urbanización.

Keywords

Public policies, informal settlements, social organizations, judicialization, urbanization.

Palavras-chave

Politicas públicas, assentamentos informais, organizações sociais, judicialização, urbanização.

territarias 40

\section{RESUMEN}

Las dos primeras gestiones del partido Propuesta Republicana en la Ciudad de Buenos Aires (2007-2015) supusieron una serie de novedades y continuidades en las políticas adoptadas para las villas y asentamientos de este distrito. A lo largo de este artículo, se buscará diferenciar algunas etapas dentro de estas gestiones y caracterizarlas. Se pondrá el énfasis en la forma en que el gobierno porteño fue transformando su perspectiva de abordaje de estas problemáticas y cómo esto se vio fuertemente articulado, en consecuencia, con un creciente interés por la presencia territorial - tanto desde las instituciones estatales como de las redes políticas- en estos barrios. A su vez, se remarcará la importancia que la judicialización de los conflictos villeros tuvo en este periodo. Este trabajo presenta algunos avances parciales de las investigaciones de los autores, para las cuales se utilizó un abordaje metodológico-cualitativo, que supuso la realización de entrevistas semiestructuradas y en profundidad y el relevamiento exhaustivo de documentación de diferentes fuentes.

\section{ABSTRACT}

The two first periods of government of the Propuesta Republicana party in the city of Buenos Aires (2007-2015) entailed a set of ruptures and continuities in the policies adopted for the informal settlements of this district. The purpose of this article is to differentiate some phases within the period analyzed and to characterize them. The emphasis will be in inquiring into the ways the government of the city transformed its approach perspective to the subject and how this was strongly articulated, in consequence, with an increasing interest in having a territorial presence -institutionally as well as with political networks - in these neighborhoods. Furthermore, the importance of the growing judicialization of urban conflicts in the district will be described. This paper presents some of the partial results of the researches that are being developed by the authors for which a qualitative methodological approach was used. Semi structured interviews were made and complemented with a survey of the existing documentation produced by the different organisms of the city government.

\section{RESUMO}

As duas primeiras gestões do partido Propostas Republicana na Cidade de Buenos Aires (2007-2015) supuseram uma série de novidades e continuidades nas políticas adotadas paras as vilas e assentamentos deste distrito. Ao longo deste artigo buscaremos diferenciar algumas etapas dentro destas gestões e caracterizá-las. Poremos a ênfase na forma em que o governo portenho foi transformando sua perspectiva de abordagem destas problemáticas e como isto viu-se fortemente articulado, em consequência, com um crescente interesse pela presença territorial -tanto desde as instituições estatais quanto das redes políticas- nestes bairros. À sua vez, remarcaremos a importância que a judicialização dos conflitos de vilas teve neste período. Este trabalho apresenta alguns avanços parciais das pesquisas dos autores, para as quais se utilizou uma abordagem metodológica qualitativa que supôs a realização de entrevistas semiestruturadas e em profundidade e o relevamento exaustivo de documentação de diferentes fontes. 


\section{Introducción}

Hacia fines de 2015, el ingeniero Mauricio Macri finalizó su segundo mandato de gobierno al frente del Poder Ejecutivo de la Ciudad Autónoma de Buenos Aires (CABA). En términos generales, su gestión se caracterizó por priorizar las áreas de la política urbana que generaban alta visibilidad, adoptando una perspectiva neoliberal, no exenta de sesgos heterodoxos.

La "cuestión de las villas" en la Ciudad ha sido tema de debate desde el mismo surgimiento de algunos asentamientos hacia principios del siglo $\mathrm{Xx}$, aunque es durante las últimas décadas que el tema ha cobrado mayor relevancia en la escena pública. Su feroz erradicación, producida por la última dictadura militar (1976-1983), así como la relativa tolerancia que mostró el Estado local al crecimiento de las villas a partir del ciclo democrático y la distinta intensidad de programas urbanos desarrollados desde los inicios de la década del noventa en el distrito, constituyeron modos de abordaje y respuesta del Estado a la cuestión.

La declaración de la Ciudad de Buenos Aires como autónoma en 1994 - distrito especial que pasaría a ser asimilable a una provincia-, y la promulgación de su propia Constitución en 1996, incorporó a esta normativa el derecho a una "vivienda digna" y la radicación de la población viviendo en asentamientos ${ }^{1}$, lo que cristalizó normativamente las luchas desarrolladas por el movimiento villero durante décadas. A partir de este momento, los avances en la reurbanización e integración urbana de estos han sido disímiles y fragmentarios, aunque constantes a lo largo del tiempo, hasta la irrupción, a partir de fines del año 2007, de la gestión del Partido Propuesta Republicana (PRO). Este hecho constituyó un cambio importante en la forma en la cual la política pública abordó la cuestión. En este sentido, se consolidó un abordaje pragmático, con una clara intencionalidad de desaliento de las expectativas de ascenso habitacional de los habitantes de las villas de la ciudad.

Las gestiones de Mauricio Macri al frente del Poder Ejecutivo de la CABA contribuyeron al empeoramiento de las condiciones de habitabilidad. De hecho, si se analiza el periodo intercensal (20012010), se observa que ha aumentado el número de personas que vivían alquilando piezas en inquilinatos u hoteles, y se había registrado un aumento de los hogares en condición de hacinamiento. También vale destacar que las cifras censales señalaban un incremento del $51 \%$ de los inquilinos respecto al año 2001.

En relación con las villas, durante las gestiones de Mauricio Macri, su población creció aceleradamente. Si bien, se trataba de un proceso que había comenzado anteriormente, el bajo rendimiento que tuvo la gestión para satisfacer la demanda habitacional reforzó, por acción u omisión, este proceso. Según el censo nacional del año 2010 (INDEC, 2010), casi un 6\% (163587 personas) de la población de la ciudad vivía en villas, núcleos habitacionales transitorios (NHT) o asentamientos, lo que implicaba un aumento del $52 \%$ respecto al año 2001 .
${ }^{1}$ El texto completo del Artículo 31 establece: " $\mathrm{La}$ Ciudad reconoce el derecho a una vivienda digna y a un hábitat adecuado. Para ello: resuelve progresivamente el déficit habitacional, de infraestructuray servicios, dando prioridad a las personas de los sectores de pobreza critica y con necesidades especiales de escasos recursos. Auspicia la incorporación de los inmuebles ociosos, promueve los planes autogestionados, la integración urbanisticay social de los pobladores marginados, la recuperación de las viviendas precarias y la regularización dominial y catastral, con criterios de radicación definitiva" (Constitución de la Ciudad de Buenos Aires, 1996)

territarias 40 229 
La información oficial más actualizada es la brindada por la Secretaría de Hábitat e Inclusión (SECHI, 2013), en la que se afirmaba que la cantidad de habitantes de las villas y asentamientos en el distrito era de 275000 personas, cifra que no incluía a los vecinos de varios asentamientos - Rodrigo Bueno, La Carbonilla y Playón de Chacarita-. A la vez, como afirma un documento de la Asociación Civil por la Igualdad y la Justicia (ACIJ, 2016), un relevamiento realizado por la ONG “Techo" durante el año 2013 estimó la población total en asentamientos informales de la ciudad en el orden de los 73300 hogares, es decir, 286000 habitantes aproximadamente.

El presente artículo intenta dar cuenta de los distintos momentos de la política pública de las gestiones del Partido Propuesta Republicana (PRO) en relación con las villas y asentamientos. En un primer apartado, se buscará presentar las características principales de la política urbana del PRO en la ciudad y, particularmente, aquellas vinculadas con el área de hábitat y vivienda, lo que permitirá presentar algunos puntos para definir el modelo de ciudad impulsado por esta gestión y el lugar de los sectores populares en ella. En el segundo apartado, se distinguirán tres momentos que pueden diferenciarse en la política del PRO hacia las villas de la ciudad, remarcando sus especificidades y continuidades a lo largo de los ocho años de gestión. En tercer lugar, se buscará remarcar el rol del poder judicial en la conflictividad villera en el distrito, enmarcándolo en un proceso más amplio de creciente judicialización y destacando un proceso en el cual se observan algunos de los efectos de lo descrito. Por último, se esbozarán algunas reflexiones finales.

$\mathrm{El}$ artículo intenta brindar un balance de la política hacia las villas del gobierno porteño durante el periodo 2007-2015, dando cuenta de las transformaciones de estas en relación con las resistencias que la gestión se encontró desde un primer momento, la construcción de redes territoriales propias, los efectos hacia adentro y afuera de los barrios de las distintas iniciativas de gobierno y los resultados que lograron concretamente en estos territorios. En términos teórico-políticos, como base, se considera a las villas como parte de la ciudad sin status de ciudad (Cravino, 2008), con derecho a su radicación definitiva, a condiciones de habitabilidad satisfactorias, un hábitat adecuado y su integración social y urbana al resto de la ciudad.

En términos metodológicos, el presente artículo posee como hipótesis que las políticas desarrolladas en los distintos momentos por las gestiones frente al Gobierno de la Ciudad de Buenos Aires de Mauricio Macri (2007-2015) han colaborado al desaliento de las expectativas de la población villera de tener una trayectoria habitacional ascendente. Las variables de análisis son las políticas del Poder Ejecutivo en relación con el sector, la conflictividad urbana en villas, y el rol que ha cumplido el Poder Judicial en la cuestión.

$\mathrm{El}$ análisis fue elaborado con base en fuentes secundarias - diarios nacionales, 
locales e informes de distintas dependencias del Estado de la Ciudad-, así como también con fuentes primarias - entrevistas semiestructuradas realizadas a habitantes de villas, funcionarios, militantes sociales y políticos-, desarrolladas en el marco de investigaciones específicas de los autores. También resulta pertinente aclarar que mucha de la información ha sido recabada en distintas instancias de trabajo de la Legislatura de la Ciudad de Buenos Aires por uno de los autores, que se desempeña allí como asesor.

\section{La política de vivienda y hábitat del Gobierno de Mauricio Macri}

Durante la gestión de Mauricio Macri al frente del Ejecutivo de la CABA (20072015), ha existido un proyecto de ciudad excluyente, aunque no siempre la política pública estuvo caracterizada por la retirada del Estado o el vaciamiento de lo público bajo la lógica del neoliberalismo más clásico. Se llevaron adelante formas más complejas y cotidianas, en las que primó una tendencia hacia la expulsión de los sectores populares de la ciudad, no solo por coerción violenta, sino también por el mercado.

Conceptualmente, se podría afirmar que la gestión tuvo un claro signo neoliberal, aunque su política se ejecutó con un carácter pragmático y heterodoxo. Esta ha impulsado una estetización del espacio público por sobre las condiciones urbanas y habitacionales de la población de la CABA, con lo que se expresa una clara concepción del orden y el sujeto urbano legítimo e ilegítimo, priorizándose la ejecución de obras de alta visibilidad y rédito político.

En otros trabajos (Palombi, et al., 2015), se ha remarcado la importancia de estudiar la política de desarrollo urbano y vivienda llevada adelante por la gestión PRO en relación con el modelo de ciudad que propone y los entramados sociales urbanos sobre los que se despliega. De esta manera, la gestión ha dividido al territorio en mosaicos de intervención, para los cuales posee diversas estrategias de acción. Dentro de la política urbana, ocupan un lugar preponderante las iniciativas tendientes a la recualificación y renovación urbana desarrolladas principalmente en el sur de la CABA y, en menor medida, en la zona norte.

Estas políticas se han desarrollado por medio de distintos instrumentos de intervención. Uno de los más utilizados fue la creación de distritos "creativos" en los que se brindan rebajas impositivas y créditos a capitales privados para promover el desarrollo temático de distintas ramas productivas y de servicios, acompañando esta política con el desarrollo de obras de mejoramiento de infraestructura y espacio público $^{2}$ (Ministerio de Economía y Finanzas de la Ciudad Autónoma de Buenos Aires, s.f.). Esta política, a pesar de que no en todos los casos tuvo el desarrollo esperado, ha redundado en el incremento de los precios del suelo (Thomasz, 2016), el consecuente desplazamiento de sectores
${ }^{2}$ Esta informa bttp://www. buenosaires.gob.ar/areas/ produccion/distrito_tecno/ territarias 40 231 
${ }^{3}$ La politica de distritos se llevó adelante en los barrios que contaban con una cantidad importante de suelo vacante por su conformación urbana - el "Distrito de las Artes" en la zona de La Boca, Barracas, San Telmo, donde se buscó consolidar un área artístico-turística; el "Distrito Tecnológico" en Parque Patricios y Nueva Pompeya, al que se trasladó la Jefatura de Gobierno de la Ciudad; el "Distrito Audiovisual" en el barrio de Colegiales; el "Distrito del Diseño" en la zona de Barracas y, recientemente el "Distrito del Deporte" en Villa Lugano y Villa Soldati-o por cuestiones puntuales (como la obra inconclusa de la Autopista 3 en la zona de Villa Ortúzar y Villa Urquiza, donde se construyó el Barrio Parque Donado Holmberg).

El caso particular de la politica de renovación urbana del sector 5 de la ex AU3 (trayecto que va del barrio de Saavedra hasta Chacarita) presenta para la gestión local una forma compleja de generar suelo urbano de muy buena calidad mediante la expulsión no coercitiva de muchos de sus habitantes (estimados en 450 familias en 2009). La sanción de la Ley 3396 en el 2010-en un contexto

\section{territarios 40}

populares y la apropiación por parte de capitales privados de cuantiosas plusvalías urbanas a partir de las sustanciales rebajas impositivas ${ }^{3}$.

Las iniciativas desarrolladas más arriba contrastan fuertemente con la política llevada adelante por la gestión en el área de hábitat y vivienda. A nivel institucional se produjo una fuerte desarticulación y fragmentación de lo existente hasta el momento y su rearticulación en nuevos organismos. En primer lugar, se puede dar cuenta de una división y —algunas veces - superposición de funciones entre seis organismos: la Dirección General Inmediata (Ministerio de Desarrollo Social), la Corporación Buenos Aires Sur (CBAS), el Instituto de la Vivienda (IVC), la Unidad de Gestión e Intervención Social (UGIS), la Secretaría de Hábitat e Inclusión (SECHI) y la Unidad Ejecutora ex Au3. Este cambio generó una profundización de la focalización de la política villera y la falta de iniciativas integrales, al mismo tiempo que trajo aparejada una dificultad para seguir la ejecución de cada uno de los programas. A pesar de que formalmente debía constituirse como una instancia articuladora para la intervención de villas y asentamientos, la creación de la SECHI, a fines del 2011, tuvo poco efecto en ese sentido. Por el contrario, se crearon nuevas dependencias y programas que supusieron la consolidación de un andamiaje institucional funcional al desarrollo de iniciativas parciales, enfocadas en garantizar niveles de gobernabilidad y atender emergencias.
Unas características destacadas en la evolución del área de hábitat y vivienda fue la casi permanente reducción y subejecución presupuestaria. El presupuesto general disminuyó de 3,67\% del total en el año 2008 a 2,63\% para el ejercicio 2015 , y los niveles de ejecución habían llegado acerca del $60 \%$ promedio para ese mismo año. Respecto al Instituto de la Vivienda, este pasó de tener una participación del $2,73 \%$, en el gasto total de la ciudad en el año 2011, a un $1,34 \%$ para el año 2015 (Ministerio de Hacienda GCABA, s.d.). También existió un proceso de desfinanciamiento del "Programa de Radicación, Integración y Transformación de Villas, Asentamientos y NHT" (PRITV) perteneciente al Instituto de la Vivienda (IVC).

La reducción y subejecución presupuestaria implicó una baja performance en la construcción de soluciones habitacionales durante el periodo 2007-2015. En este sentido, los programas y áreas que abordaban la emergencia habitacional han tenido más peso que aquellos vinculados al otorgamiento de soluciones habitacionales definitivas. A la vez, la política de priorizar lo "visible" se manifestó con especial intensidad en el área: tanto los "Portales Inclusivos"4 que llevó adelante la SECHI, como el maquillaje urbano aplicado en la pintura de fachadas, especialmente en los bordes de las villas y algunos conjuntos habitacionales, dan cuenta de la prioridad que ha tenido "lo visible" dentro de la política de vivienda. 
En relación con la atención de la emergencia habitacional por sobre el otorgamiento de soluciones habitacionales definitivas, se observó dentro de las villas, con la creación de cooperativas de mantenimiento y limpieza, desarrolladas por la Unidad de Gestión e Intervención Social (UGIS), que se ocuparon básicamente de arreglos eléctricos, en general precarios, el destape de caños cloacales, tareas de limpieza, entre otras. A la vez, el Instituto de la Vivienda (IVC) dentro de los conjuntos habitacionales bajo su órbita ${ }^{5}$ llevó adelante una política similar (cambio de luminarias, pintura de frentes, mantenimiento de pluviales etc.). También, el altísimo grado de ejecución de la Dirección de Atención Inmediata del Ministerio de Desarrollo Social, dependencia que brindaba subsidios habitacionales por un plazo de seis meses para alquilar una habitación, da cuenta de la manera en la cual la gestión tuvo que abordar las cuestiones más urgentes que podían generar un grado importante de conflictividad.

Un elemento también importante a la hora de realizar un balance sobre el área durante las gestiones de Mauricio Macri es el énfasis puesto en las políticas de regularización dominial, más allá del mejoramiento de las condiciones de habitabilidad existentes, dentro de las villas, asentamientos y conjuntos urbanos. Si bien, en el caso de las villas solo lograron escriturar algunos pobladores, la gestión consolidó un cuerpo normativo que empeoró las ya precarias condiciones habitacionales de los habi- tantes de los asentamientos. En el caso de los conjuntos habitacionales urbanos, la política de regularización tuvo una escala mucho mayor que en las villas, lográndose escriturar unidades habitacionales sin realizar ningún tipo de puesta en valor, aun en los conjuntos que requerían intervenciones de carácter estructural.

Otro aspecto relevante es el bajo grado de ejecución de los fondos nacionales de vivienda. Al momento de asumir la primera gestión de Mauricio Macri, existían 2120 viviendas en ejecución en la ciudad bajo esta modalidad, de las cuales todavía faltaba terminar 726 al año 2013. A la vez, de los 963 mejoramientos de vivienda comprometidos hacia fines del año 2007, a principios de 2013 todavía habían quedado pendientes 577 obras $^{6}$ (Consejo Nacional de la Vivienda, 2014).

Finalmente, también se evidenció un bajo grado de cumplimiento de las diversas sentencias judiciales vinculadas con temas de hábitat y vivienda. En relación con la llamada causa "Mendoza", que dictaminó el saneamiento de la cuenca de los ríos Matanza-Riachuelo, se observó una baja ejecución de las obras comprometidas y el reiterado incumplimiento de los plazos estipulados. Luego de 8 años de dictada la sentencia por la Corte Suprema de Justicia de la Nación, en el año 2015, el Gobierno de la Ciudad Autónoma de Buenos Aires solamente había relocalizado al $23 \%$ de las familias residentes en los márgenes del río ${ }^{7}$ (Diario Nueva Ciudad, 2018). de desalojos administrativos compulsivos-incorporó la posibilidad de entrega de subsidios (prestaciones no reintegrables) a muchos de los vecinos que habitaban en el sector nombrado, alternativa que no figuraba en la Ley 324 (que en el año 1999 habia creado el Programa de Recuperación de la Traza de la Ex AU3). Muchos pobladores, luego de años de incumplimientos, eligieron este mecanismo frente a seguir esperando una respuesta definitiva. De esta manera, se logró la desocupación de muchas parcelas que fueron subastadas a precios de mercado. El cambio de zonificación de estas, y las obras complementarias que realizó el Estado transgrediendo la propia Ley 3396, significaron una apropiación fenomenal de rentas a favor de empresas privadas ligadas en muchos casos a la gestión del PRO.

${ }^{4}$ Los "Portales Inclusivos" eran sitios dedicados a la práctica de deportes, recreación y al desarrollo de cursos de formación en oficios. Estos predios estaban localizados en sectores de las villas que pudieran ser vistos por el resto de los habitantes de la ciudad.

${ }^{5}$ El Instituto de la vivienda posee bajo su órbita 16 conjuntos habitacionales construidos durante las

territarias 40 
últimas décadas por la ex Comisión Municipal de la Vivienda, muchos de los cualesse encuentran degradados. También resulta importante resaltar que existen diversas leyes generales y particulares que obligan al Estado a solucionar diversos aspectos de estas que han sido sistemáticamente incumplidas.

${ }^{6}$ Elaboración propia en base a datos del Consejo Nacional de la Vivienda www. cnvivienda.org.ar

${ }^{7}$ Elaboración propia en base a datos de Gobierno de la Ciudad y ACUMAR.

${ }^{8}$ El conflicto del Parque Indoamericano se desarrolló hacia mitad de diciembre de 2010 y constituyó una de las tomas de tierra más importantes de las últimas décadasen la Argentina. Durante aproximadamente 10 dias habitantes de la zona sur de la ciudad construyeron casillas en un parque público que se encontraba en situación de abandono, llegando a contabilizarse aproximadamente 13000 personas en el momento de mayor conflictividad. Este finalizó con un acuerdo por medio del cual se le daría solución a esta población, que hasta el día de boy no ha sido cumplida por parte del Estado.

${ }^{9}$ Nótese que el dictamen que

territarias 40

\section{Distintos momentos de abordaje de la cuestión villera en la gestión PRO}

\subsection{El aprendizaje de la gestión: del discurso y la práctica del desalojo a la construcción de herramientas de intervención (2007-2010)}

Con respecto a la gestión de villas y asentamientos, el discurso y la práctica del gobierno de Mauricio Macri fueron sumamente ambiguos y contradictorios. La primera etapa que distinguimos se extiende desde que la asunción del PRO a la Jefatura de Gobierno en diciembre de 2007 hasta los sucesos del Parque Indoamericano ${ }^{8}$ (2010).

En la campaña electoral, Mauricio Macri anunció que de ganar impulsaría la radicación de las villas de la ciudad, con excepción de la Villa 31-31 bis Retiro (Página 12, 2007), ubicada cerca del microcentro y de la villa Rodrigo Bueno, situada en la Reserva Ecológica de la ciudad, que serían erradicadas (Dandan, 2007). Este discurso y accionar fue cambiando cuando el PRO asumió la gestión de la ciudad, llegándose a la sanción por unanimidad en la Legislatura de la ciudad en el año 2009 de la Ley 3343 de urbanización de la Villa 31-31 bis ${ }^{9}$, aunque la decisión de erradicar la villa Rodrigo Bueno, por su parte, no cambión ${ }^{10}$ durante toda la gestión.

En términos generales, la política hacia los asentamientos en esta primera gestión del PRO fue pragmática y fragmentada, contemplando las correlaciones de fuerza y el contexto en el caso de cada barrio (Cravino et al., 2013). Esto significó que las iniciativas fueron de acuerdo con la orientación y capacidad de las organizaciones villeras, las presiones de los pobladores vecinos a los barrios y del mercado inmobiliario, así como también en relación con las pujas políticas dentro del mismo oficialismo (Cravino \& Palombi, 2015). Este periodo se podría caracterizar como de aprendizaje, en el marco de que el partido gobernante no tenía arraigo político en este tipo de barrios. Por otra parte, era la primera experiencia de gobierno del PRO $\mathrm{y}$, sin duda, la cuestión de las villas era una problemática compleja y no prioritaria, por lo menos en un primer momento.

Durante esta etapa se creó, en el año 2008, la Unidad de Gestión e Intervención Social (UGIS), dependencia que en los hechos comenzó a funcionar a partir del año 2009 y que, como más adelante se desarrollará, cobraría mayor importancia en los ejercicios siguientes. Si bien, la función de este organismo debía ser planificar la urbanización de villas, asentamientos y NHT, en la práctica se abocó a la atención de emergencias y algunas obras de mejoramiento, como la contratación de empresas de prestación de servicio de camiones atmosféricos y de higiene urbana, entre otras cuestiones. También sería el organismo responsable de las obras y el servicio de mantenimiento de las redes eléctricas y cloacales. A la vez, esta dependencia debía realizar las tareas de remodelar y/o construir algunas viviendas en caso de riesgo de vida de sus residentes, pero en los hechos 
prácticamente no intervino en la cuestión. Finalmente, el organismo tenía un área de fortalecimiento socio-comunitario y de articulación con juntas vecinales y cuerpos de delegados, instancias de organización y participación de los pobladores de los distintos barrios.

Por otra parte, durante esta primera etapa se erradicaron algunos de los nuevos asentamientos urbanos que habían surgido durante la década anterior. Estos barrios de dimensiones pequeñas estaban emplazados en intersticios urbanos, caracterizándose por estar construidos con materiales precarios, siendo sus pobladores en su mayoría recuperadores urbanos ('cartoneros'). Se destaca el desalojo de "Villa Hollywood" (Infobae, 2010)11 por parte del Ministerio de Ambiente y Espacio público, el Asentamiento Caballito o Morixe (Asociación Civil Caballito Puede, $2009)^{12}$ con el acompañamiento del mismo Jefe de Gabinete de ese entonces, Horacio Rodríguez Larreta, y el de Villa Topo en el Barrio de Constitución (Clarin, 2008) ${ }^{13}$.

Como se afirmó anteriormente, esta primera etapa de la política pública de la gestión se extiende hasta la denominada “Toma del Parque Indoamericano". Este hecho constituyó uno de los conflictos de mayor relevancia pública en los últimos años en la Argentina. Esta toma de un sector de un parque desocupado en la Comuna 8, que se extendió durante varias semanas junto con una serie de conflictos similares, puso de manifiesto las crecientes dificultades en el acceso a una vivienda y suelo urbano en la Región Metropolitana de Buenos Aires ${ }^{14}$. Creemos que este hecho generó un reacomodamiento hacia dentro de la política pública del PRO, lo que generó una fuerte preocupación respecto a la gestión de la conflictividad urbana y, por ende, de la gobernabilidad de las villas de la ciudad.

\subsection{La consolidación del 'maquillaje urbano' como política de gobernabilidad (2010-2012)}

Luego del conflicto al que se hizo referencia anteriormente, la política hacia las villas y asentamientos profundizó su carácter de atención de emergencias e intervenciones superficiales, prestando atención a la construcción y refuerzo de una trama política que fortaleciera la gobernabilidad de la gestión. A partir de esta etapa, los objetivos que podrían cumplir los habitantes de las villas estarían dados por el mejoramiento de las fachadas y del espacio público y, en una etapa posterior, la regularización dominial salvaje de "lo construido". Estas iniciativas, que en otros artículos (Cravino \& Palombi, 2014) se han denominado de 'maquillaje urbano', consistieron en la entrega de recursos a cooperativas, en mayor medida manejadas clientelarmente, para que pintaran las fachadas de las viviendas o realizaran obras de mejoramiento en el espacio público de las villas, sin resolver el acuciante problema del déficit de servicios públicos urbanos y el hacinamiento. De esta manera, la UGIS celebró múltiples convenios con distintas cooperativas, muchas de las cuales estaban integradas por vecinos truir participativamente en la Mesa de urbanización del barrio no fue tratado en la Legislatura Porteña por años, por lo que la Ley 3343 nunca se cumplió efectivamente.

${ }^{10}$ El conflicto por la urbanización de la villa Rodrigo Bueno se haya judicializada desde el año 2005 en el cual los vecinos presentaron un recurso de amparo ante la orden de desalojo del barrio. Mauricio Macri apeló a ese amparo como Jefe de Gobierno, y el caso continúa siendo altamente conflictivo. Existe un interés central por parte del Estado de desalojar el asentamiento para permitiv el desarrollo de un megaproyecto inmobiliario por parte de IRSA. - Esta información está ampliada en el libro Las trampas de la naturaleza de María Carman (2011)-.

${ }^{11}$ Ver http://www.infobae. com/2010/11/23/548514desalojan-los-habitantesvilla-hollywood-palermo/

12 Ver http://www.cabalitopuede.com.ar/newspost.php?id=52

${ }^{13}$ Ver http://edant.clarin. com/diario/2008/06/11/ laciudad/h-01691461.htm

14 Para un análisis del conflicto puede consultarse Cravino (2014)

tersitarios 40

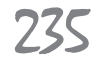


15 Organismo creado mediante el Decreto N. ${ }^{\circ} 660$ $y$ modificadas su funciones mediante el Decreto N. ${ }^{\circ} 149$ teniendo como objetivo coordinar las acciones de las diferentes áreas del Gobierno de la Ciudad de Buenos Aires para una intervención integral en las villas y barrios vulnerables. Las funciones que debia desarrollar según esta normativa fueron proyectos de urbanización (ordenamiento del suelo y servicios básicos), urbanismo social (intervenciones físicas de mejoramiento de los barrios), proyectos sociales, culturales, deportivos, educativos, de productividad, de medioambiente, salud y género.

${ }^{16}$ Esta iniciativa se encontraba, en la primera etapa de gestión descrita, asignado al Ministerio de Ambiente y Espacio Público, ocupándose de la realización de obras mediante el convenio con distintas cooperativas.

${ }^{17}$ Para un desarrollo particular del discurso y la construcción del PRO como fuerza politica puede consultarse Vommaro et al. (2015).

\section{territarias 40}

de los barrios, lo que la convirtió en un organismo con fuerte impronta política. El organismo presentó grados de ejecución presupuestarias mayores al 95\% durante estos años y fuertes redireccionamientos de partidas de dinero a su favor.

Otro de los instrumentos relevantes que emergió en esta etapa fue la Secretaría de Hábitat e Inclusión (SECHI $)^{15}$. Este organismo desarrolló la experiencia de los "Portales de Inclusión" - construcción de espacios públicos orientados a lo deportivo y el desarrollo de microemprendimientosen asentamientos de gran escala y complejidad como la Villa 1-11-14 (Bajo Flores) y la Villa 20 (Villa Lugano), aunque también en otros de menor tamaño como la Villa 6 (Cildañez) y el barrio Los Piletones. La entidad también se ocupó del tendido de algunos servicios y otras acciones de baja intensidad, como el "diagnóstico participativo para la producción del hábitat social", jornadas para el fortalecimiento de capacidades y planificación social. También la SECHI gestionó el programa de mejoramiento de la Villa 31 y 31 bis $^{16}$.

Por otra parte, si bien este organismo debió cumplir un rol articulador de la política dentro del Ministerio de Desarrollo Económico, en los hechos funcionó más como una unidad de proyectos especiales que como secretaría de coordinación de la política en villas y asentamientos. Se podría afirmar que algunas de las acciones encaradas han tenido cierto éxito en los barrios donde se ha implementado, aunque su tarea ha sido limitada y focalizada.
Resulta importante remarcar que este tipo de intervenciones, en general realizadas en nombre de una política de cercanía con los vecinos, con rasgos de voluntariado, han generado redes territoriales extendidas sobre las cuales construyó la gestión su sustento político partidario de cara a las elecciones legislativas locales, proyectándose a las nacionales en 2015, cuando el jefe de gobierno se presentó como candidato a presidente del país ${ }^{17}$. Esto implicó una presencia cotidiana de gran cantidad de agentes estatales en los barrios, lo que ha demostrado que la política pública del partido PRO no supuso una retirada del Estado, enfoque asociado con el neoliberalismo, sino una presencia territorial de diferente tipo. En particular, merece destacarse que este despliegue de funcionarios se hizo sin priorizar la interlocución con los referentes villeros elegidos por los propios vecinos de estos barrios. Se puede afirmar, por lo tanto, que se desarrolló un estilo de construcción política con un discurso referido a la 'no-política'.

\subsection{La invención de la \\ "regularización salvaje" y la política de "acupuntura urbana" (2012-2015)}

Durante el periodo 2012-2015 la gestión apostó fuertemente al avance de los procesos de regularización dominial, más allá de la consolidación de los principales rasgos de las políticas de la etapa anterior. Según entrevistas realizadas a funcionarios, hacia fines de 2015, se habían entregado 180 
escrituras en la Villa 19-INTA, e inicio de trámite en el Barrio Piletones.

Las obras complementarias al proceso de regularización dominial fueron llevadas adelante en esta etapa por el Programa Prosur Hábitat de la Corporación Buenos Aires Sur. Los casos de estudio que hemos relevado (Villa 19, Villa 6, barrio Los Piletones y Villa 20), así como también lo afirma un informe realizado por la Asesoría General Tutelar (2014), nos demuestran que el accionar de este programa fue profundamente deficiente en tanto política de reurbanización e integración de las villas y asentamientos de la zona sur. Solamente se encuentra dentro de este la realización de algunas pocas obras de infraestructura —cloacas principalmente-, la apertura de 5 o 6 cuadras de distintas calles y el mejoramiento de espacios públicos. Esta dependencia denominaba a estas obras dentro del paradigma de "acupuntura urbana", término que grafica muy bien el carácter focalizado de las iniciativas.

El proceso llevado adelante en la Villa 19-INTA, guiado por la Ley 4232/12 que establecía las bases para su 'urbanización', fue mostrado como el paradigma de la política de la gestión. Más allá de respetar algunas cuestiones mínimas enunciadas en la ordenanza $44873 / 91^{18}$, esta ley presentaba una flexibilización importante del código de planeamiento urbano y de edificación respecto a la regularización de lo existente $^{19}$. Luego, con la sanción de las Leyes 5197/14 (Villa Cildañez) y 5235/14 (Villas de la comuna 8 , con excepción de la Villa 20) se buscó, en mayor medida que en el caso anterior, legalizar lo previamente construido ${ }^{20}$. La Villa 19-INTA ya había sido objeto de diversas intervenciones a partir de mediados de la década del noventa (Arqueros, 2016) - previo a los procesos de densificación e inquilinización-, por lo que la forma de los polígonos, el ancho de las calles y demás ya se encontraban en proceso de consolidación ${ }^{21}$. En los otros casos, excepto la villa 6 Cildañez, los barrios no habían sufrido intervenciones de magnitud anteriormente, por lo que se requería un verdadero proceso de reurbanización e integración social y urbana para lograr su consolidación, aspecto que la gestión encabezada por Mauricio Macri no estuvo dispuesta a llevar adelante.

En un primer momento, este proceso de regularización dominial de las villas de la Comuna 8 de la CABA fue incorporado dentro de un capítulo del denominado “Master Plan de la Comuna 8", en el cual se contemplaba también el desarrollo del "Distrito del Deporte", desarrollos residenciales para clases medias y la construcción de una Villa Olímpica para los Juegos Olímpicos Juveniles del 2018, entre los emprendimientos destacados. De esta manera, la política de regularización dominial era presentada por los legisladores del partido gobernante como la "cara social” de este proyecto que se proponía la recuperación de suelo en condiciones de vacancia para el desarrollo del mercado inmobiliario. Luego, en el marco de una fuerte resistencia de organizaciones y bloques legislativos, se optó por hacer votar el proyecto en la Legislatura de la Ciudad
${ }^{18}$ El lote minimo debía ser de $72 \mathrm{~m}^{2}$ estipulado en esta (aunque admite una tolerancia de +-10\%), un FOS de $65 \%$ para construcciones futuras y un ancho de sendas de $4 m$ y de las calles de $10,5 \mathrm{~m}$.

${ }^{19}$ En este sentido, se suprimian los patios auxiliares, asi como también las divisiones minimasinternas de los lotes y su registro catastral. A la vez, se autorizaba a la dirección general de interpretación urbanistica a dictaminar sobre aspectos no previstos de la ley.

${ }^{20}$ En este sentido, existen excepciones para las medidas del lote mínimo, se permite construir toda la superficie del lote, sin tener en cuenta los patios auxiliares a futuro y se reducen las calles a $8 m$ y $2 m$ en el caso de las sendas (con una tolerancia de +- 10\%). A la vez, se autoriza a la Dirección general de interpretación urbanistica a dictaminar sobre aspectos no previstos.

${ }^{21}$ Di Virgilio et al. (2012) afirman que a mediados de la década del noventa comenzaron a desarrollarse las primeras obras de urbanización en el barrio INTA. Los autores explican cómo, en los últimos 15 años, el barrio habia tenido un importante proceso de mejora: todas las calles principales

tersitarios 40

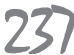




\section{$\Longleftarrow$}

habian sido pavimentadas; la mayor parte de los pasillos 12 habian desaparecido; y se instalaron y conectaron las viviendas a los servicios de agua de red y cloaca, entre otras cuestiones.

\section{territarias 40}

solo con el cambio de normativa para los asentamientos, la creación del "Distrito del Deporte" y la construcción de la Villa Olímpica.

Resulta importante analizar cuál es la concepción de "regularización dominial" que se encuentra presente en estas intervenciones, ya que puede entenderse de distintas formas. En un extremo, se ubica la regularización dominial del suelo urbano ocupado, tal como se realizó en Perú a partir de las ideas planteadas por el economista Hernando De Soto (1987). Esta suponía legalizar lo existente sin tener en cuenta las condiciones urbanas donde estaba implantado el barrio o la vivienda ni las características edilicias del inmueble o el cumplimiento de la normativa urbana. En el otro, constituye la etapa final de un ciclo de micro intervenciones en los asentamientos populares que se concentra en la integración de la trama urbana al resto de la ciudad (por lo general por medio de apertura de calles), su equiparación en términos de provisión de infraestructura urbana (conexiones y medidores individuales), el mejoramiento de las viviendas, así como también la creación de espacio público y la instalación de otros equipamientos necesarios (escuelas, centros de salud y otros equipamientos barriales). En un caso es el inicio y fin de un proceso, que se cierra en la posesión de la escritura del inmueble existente, y en el otro es la culminación de un procedimiento de mejoramiento barrial y habitacional (Cravino \& Palombi, 2014).
Retomando el debate latinoamericano, existe una amplia literatura que indica que la mera legalización de los asentamientos no resuelve el problema. Como afirma Edesio Fernandes (2008), estas políticas no se basan en la comprensión de la dinámica de la informalidad urbana, es decir que existen múltiples procesos a los cuales no logra dar respuesta. En particular, en la Ciudad de Buenos Aires este procedimiento se da en un marco de crecimiento exponencial de la población viviendo en este tipo de asentamientos, a la vez que tampoco toma en cuenta el empeoramiento de las condiciones de infraestructura y hacinamiento, así como la cantidad creciente de inquilinos en estos barrios.

Tampoco la gestión realizó ningún tipo de estudio en relación a la factibilidad en la economía familiar de los costos de la regularización (pago del suelo, servicios e impuestos, entre otros). Resulta evidente que se renunció totalmente a la capacidad de fiscalización por parte del Estado. De esta forma, el acento estuvo puesto en la forma geométrica de los polígonos correspondientes a las manzanas, quedando la compleja división interna de las construcciones y lotes "blanqueada", legalizándose lo existente, implicando que los conflictos suscitados o a suscitarse a futuro entre vecinos pasaban a convertirse en discrepancias entre privados.

Creemos que resulta importante relacionar los intentos de regularización dominial llevados adelante por el gobierno local con la caracterización que se realizó anteriormente respecto a las distintas 
políticas llevadas adelante en el área de hábitat y vivienda. En este sentido, los intentos de regularización dominial en las Comunas 8 y 9 han perseguido un objetivo político de garantizar gobernabilidad en estos territorios luego de momentos de alta conflictividad, aplicándose un criterio casi empresarial que tiene en cuenta la relación entre costo, eficiencia y resolución de problemas, es decir, pocos recursos para generar una red de gobernabilidad

Hemos visto en este apartado cómo, dentro de esta etapa, se resignificó la idea de la reurbanización de villas e integración urbana, intentando trasladarse la conflictividad a los propios vecinos, lo que generó fragmentación y una lógica en la que deben resolver ellos mismos sus problemáticas. Si bien, el proceso de regularización dominial ha tenido un carácter limitado, es importante tomarlo como un momento de la política, ya que cristaliza una intencionalidad marcada del Ejecutivo de la ciudad en estos años.

\section{Respuestas a situaciones de judicialización}

La cuestión de la judicialización de los conflictos villeros en la ciudad de Buenos Aires durante el periodo analizado resulta significativa para introducir en este artículo, ya que constituyó un campo en el que crecientemente se disputaron las políticas hacia las villas delineadas por el PRO. Diversos autores han presentado estos procesos (Arqueros et al., 2011; Bercovich \& Maurino, 2013; Delamata, Sethman,
Ricciardi, 2014; Cravino, 2016: Fainstein, 2015). Para finales de la segunda gestión del PRO, al frente de la ciudad este proceso se encontraba en declive (Cravino, 2016). Por una parte, por los pocos efectos concretos que la resolución de los conflictos por la vía judicial tuvo en el distrito más allá de sus efectos simbólicos y políticos (Bercovich, 2013; Bermúdez, Carmona Barrenechea \& Royo, 2014)y, por el otro, por el nombramiento y ascenso de jueces afines al oficialismo y el corrimiento de otros más opositores dentro del Poder Judicial de la CABA.

Estos procesos se enmarcaron en un contexto más general que se venía desarrollando desde los años ochenta en la región, vinculado con dos cuestiones interrelacionadas que se consolidaron en la década del noventa con las reformas neoliberales del Estado (Arqueros et al., 2011): por un lado, una "estandarización jurídica" que se llevó adelante para agilizar el flujo comercial y financiero y generar un clima de inversión estable. Por otra parte, la creación de una serie de recursos legales para reclamar por los derechos económicos, sociales y culturales (DESC), que generaron un novedoso campo de activismo judicial y una plataforma de reclamos de los movimientos sociales que los encontraban ahora legitimados normativamente. Además, se desarrollaron reformas constitucionales y dentro del poder judicial en sí que significaron la ampliación de los derechos económicos, sociales y culturales.

En la Argentina específicamente, a finales de la última dictadura militar se territarias 40 239 
${ }^{22}$ Expediente 31699/0 disponible en la página web del Poder Judicial de la Ciudad: http://basefuero. jusbaires.gov.ar

${ }^{23}$ Ver http://www.clarin. com/ciudades/Buscan-garantizar-entren-ambulancias-villas_0_1195080600. btml

24 Ver http://www.paginal2.com.ar/diario/sociedad/3- 125247-200905-20.html

\section{territarias 40} 240 abrió un proceso en el que los organismos de defensa de los derechos humanos utilizaron crecientemente los tribunales y discursos que invocan derechos como instrumentos para hacer política (Smulovicz, 2009). Comenzaron entonces a definirse y redefinirse políticas públicas desde la justicia y la utilización creciente de las cortes y las herramientas jurídicas por parte de las organizaciones sociales y la población en general para realizar demandas privadas y sociales.

En la CABA este proceso se acentuó durante las dos gestiones del PRO, dando lugar a varias causas de gran envergadura como el amparo presentado por el incumplimiento por parte del Gobierno de la Ciudad en la forma de realización de los comicios en las villas de la ciudad estipulada por la Ley $148^{22}$ (Delamta, Ricciardi \& Sethman, 2014), la "causa Rodrigo Bueno" por la urbanización de este asentamiento, los casos por servicios públicos como el de riesgo eléctrico en la villa 21 24 de Barracas o aquella por la garantía de la entrada de ambulancias en las villas (Clarin, 2014) $)^{23}$, la orden de clausura del depósito de autos de la Policía Federal al costado de la villa 20 por la contaminación ambiental que genera (Página 12, 2009) ${ }^{24}$, y la de la implementación de relocalizaciones de villas y asentamientos a partir de la Causa "Mendoza" y el incendio de Villa "Cartón".

El campo jurídico se conformó por lo tanto como un espacio más de la arena de disputa política (Smulovitz, 2009), transformando en cierta medida la vinculación entre los organismos estatales y las organizaciones villeras, que llevaron adelante nuevos repertorios de acción colectiva - repertorios legales como los llamó Smulovitz (2009) - que desplazaron en algunos casos las instancias tradicionales de negociación con Poder Ejecutivo porteño (Arqueros et al., 2011). La multiplicidad de operadores jurídicos, particularidad de la CABA, fue importante en el desarrollo de este tipo de conflictividad, ya que una pluralidad de actores pertenecientes tanto a ONG dedicadas al litigio de derechos humanos y al Poder Judicial de la CABA (Defensoría General de la Ciudad y Ministerio Público Tutelar de la Ciudad) tuvieron roles centrales en el acercamiento a estos territorios y el impulso de estas causas en los tribunales, en los que se ubicaron también un número de magistrados que acompañaron los litigios surgidos de las villas. El fuerte activismo judicial de este periodo, portador de un enfoque de derechos, tuvo un rol preponderante en el asesoramiento y acompañamiento de los conflictos judicializados.

\section{Consideraciones finales}

A lo largo de este artículo, se ha desarrollado un análisis general de la política hacia las villas que se desarrollaron durante las gestiones de Mauricio Macri (2007-2015) al frente del gobierno porteño, rescatando los distintos momentos, su relación con las particularidades de los diversos barrios, la conflictividad urbana y los procesos de judicialización. 
En términos generales, resulta necesario destacar como rasgo distintivo los altos niveles de pragmatismo y fragmentación en las políticas de esta área, que se expresó tanto en los programas en sí, como a nivel institucional. Además, puede observarse una primacía en el desarrollo de políticas de emergencia y maquillaje urbano en desmedro del desarrollo de soluciones habitacionales definitivas. Estas políticas significaron una importante reducción presupuestaria y subejecución de los programas relacionados con la concreción de soluciones habitacionales definitivas.

Luego de algunas iniciativas frustradas, se produjo un aprendizaje por parte de la gestión, dándole importancia a la presencia territorial en las villas y a la construcción de referentes y redes sociales y políticas propias. De esta manera, se produjeron nuevas maneras de articulación y comunicación con el Estado hacia dentro de los barrios, dejando de lado a los representantes barriales elegidos por los propios vecinos. y a los referentes históricos de cada asentamiento. Este accionar se vio también reflejado en el discurso del gobierno, que, en nombre de la no política, elogiaba a las políticas participativas y de inclusión en los barrios.

Si durante un primer momento, que se extiende desde el año 2007 hasta el 2010 , se comenzó con un discurso y una práctica orientada al desalojo de distintos asentamientos, la invención de la Unidad de Intervención y Gestión Social durante el año 2009 significó un intento de adentrarse en los barrios y comenzar a construir herramientas de intervención que lograran generar gobernabilidad y redes territoriales de diálogo con el Estado. Luego, a este primer intento se le sumó el desarrollo de las políticas de maquillaje urbano (pintado de fachadas y mejoramiento de espacios públicos) a partir del año 2010, consolidándose con la creación de la Secretaría de hábitat e Inclusión hacia el año 2012. Finalmente, la gestión complementa sus herramientas de intervención con la implementación de la regularización dominial y la llamada "acupuntura urbana" a partir del año 2013.

La dinámica entre judicialización, conflictividad y respuesta del Estado también constituyó un elemento central que incidió en muchas de las políticas. Si bien, la gestión respondió a cada una de las sentencias de manera tardía, o directamente las incumplió, esta dinámica incidió fuertemente en la política más general orientada a villas, de hecho, el magro stock de vivienda construido por la gestión debió ponerse a disposición de distintas sentencias judiciales. En este campo, el Ejecutivo también tuvo un aprendizaje, cambiándose los jueces más progresistas por otros más afines a la gestión.

Si se observa la intervención del Estado, intentando brindar una mirada complementaria en relación con los distintos momentos de implementación de la política, se puede afirmar que se trató de una política de desaliento de las expectativas de la población villera de tener una trayectoria habitacional ascendente, consolidando la situación de precariedad y debilitando los marcos de conflictividad. Queda pendiente territarias 40 241 
para un próximo trabajo estudiar el impacto que tuvo esta cuestión en los sectores organizados dentro de las villas y los referentes históricos de las mismas.

\section{Referencias}

Asociación Civil Caballito Puede (2009). Desalojo del asentamiento Morixe, Diario digital Caballito Puede. Recuperado de http://www.caballitopuede.com.ar/news-post.php? $\mathrm{id}=52$

Arqueros, S. (2016). La cuestión de la radicación de villas: una mirada desde las burocracias estatales. Cuadernos de Vivienda y Urbanismo, 9(17), 28-49.

Arqueros, S., Calderón, C., Jauri, N., Ramos, J., Vitale, P., \& Yacovino, P. (2011). Territorios y expedientes. Cuatro casos de intervenciones judiciales en la Ciudad de Buenos Aires. Ponencia presentada en las VI Jornadas de la carrera de Trabajo Social de la Universidad de Buenos Aires, Buenos Aires, Argentina.

Bermúdez, A., Carmona Barrenechea, V., Ferreira, M., Kalpschtrej, K., Logiudice, A. Peisojovich, M., et al. (2014). Procesos de urbanización de villas de la CABA. Los casos de villa 19 - INTA. Villa 20 y Los Piletones. Documento de Trabajo ${ }^{\circ}$ 19. Documentos de Trabajo del Ministerio Público Tutelar, 19, 2-109.

Carman, M. (2011). Trampas de la naturaleza. Medio ambiente y segregación en

\section{territarias 40}

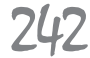

Caballito Puede (2009). Desalojo del asentamiento Morixe, Diario digital Caballito Puede. Recuperado de http:// www.caballitopuede.com.ar/newspost.php? id $=52$

Clarín (2008). Empiezan a sacar los puestos en los andenes de Constitución. Diario Clarin. Recuperado de http://edant. clarin.com/diario/2008/06/11/ laciudad/h-01691461.htm

Clarín (2014). Buscan garantizar que entren las ambulancias a las villas. Diario Clarín. Recuperado de http:// www.clarin.com/ciudades/Buscangarantizar-entren-ambulancias-vi1las_0_1195080600.html

Cravino, M. C. (2008). Vivir en la Villa. Relatos, trayectorias y estrategias habitacionales. Los Polvorines: Editorial UNGS.

Cravino, M., Ricciardi, M., \& Sehtman, A. (2013). De la programación a la administración o de los anuncios del pragmatismo: avatares de la política de villas del macrismo (2007-2011), en Bercovich, L., \& Maurino G. (coord.) Los Derechos Sociales en la Gran Buenos Aires (pp. 335-358). Buenos Aires: Eudeba.

Cravino, M. C. (org.) (2014). Derecho a la ciudad y conflictos urbanos. La ocupación del parque indoamericano. Los Polvorines: Editorial UNGS.

Cravino, M. C., \& Palombi, A. (2015). Políticas urbanas en el sur de Buenos Aires durante el gobierno de Mauricio Macri. Cuadernos de Vivienda y urbanismo 8(15), 40-51. Bogotá.

Carla Fainstein, Ariel Matías Palombi 
Cravino, M. C. (2016). Urbanización de villas y asentamientos en Buenos $\mathrm{Ai}$ res. Colección Es Nuestra la Ciudad de la Asociación Civil por la Justicia y la Igualdad, 3, 5-19.

Cravino, M. C., \& Fainstein, C. (2017). Disputas por el acceso al ambiente sano y la vivienda en la ribera del Riachuelo: derechos de los vecinos, acción de la Justicia y políticas públicas. En Cravino, M. C. (coord.), Detrás de los conflictos. Estudios sobre desigualdad urbana en la Región Metropolitana de Buenos Aires (pp. 53-114). Los Polvorines: Editorial UNGS.

Dandan, A. (2007). Los más pobres en tierras muy caras. Diario Página 12. Recuperado de http:// www.paginal2.com.ar/diario/elpais/1-88908-2007-07-30.html

Delamata, G., Sethman, A., \& Ricciardi, M. V. (2014). Más allá de los estrados... Activismo judicial y repertorios de acción villera en la ciudad de Buenos Aires en Pautassi, L. (Dir.) Marginaciones sociales en el Área Metropolitana de Buenos Aires (pp. 397-444), Buenos Aires: Editorial Biblos

Diario Nueva Ciudad (2018). Ciudad: aún resta relocalizar al 63\% de la población que vive a orillas del Riachuelo. Recuperado de https://www.nueva-ciudad. com.ar/notas/201802/36375-ciudad-aun-resta-relocalizar-al-63-dela-poblacion-que-vive-a-orillas-delriachuelo.html
Do Soto, H. (1987). El otro sendero: la revolución informal. Buenos Aires: Sudamericana.

Fernandes, E. (2008). Consideraciones generales sobre las políticas públicas de regularización de asentamientos informales en América Latina. Revista Eure, XXXIV (102), 25-38.

Infobae (2010). Desalojan a los habitantes de "Villa Hollywood" de Palermo. Diario Infobae. Recuperado de http://www.infobae. com $/ 2010 / 11 / 23 / 548514$-desalojan-los-habitantes-villa-hollywoodpalermo/

Instituto Nacional de Estadísticas y Censos (2012). Censo nacional de población, hogares y viviendas 2010. Censo del Bicentenario: resultados definitivos, Serie $\mathrm{B} \mathrm{n}^{\mathrm{O}}$ 2.-1 a ed.-Buenos Aires: INEC.

Ley 4232/12. Argentina. Legislatura de la Ciudad Autónoma de Buenos Aires. Modificación del Código de Planeamiento. 05 de julio 2012

Ley 5197/14. Argentina. Legislatura de la Ciudad Autónoma de Buenos Aires. Desafectación de Distritos. 04 de diciembre 2014

Ley 5235/ 14. Argentina. Legislatura de la Ciudad Autónoma de Buenos Aires. Promoción de las Actividades de la Producción e Industria Deportiva en la Ciudad Autónoma de Buenos Aires. 11 de diciembre 2014

Merlinsky, M. G. (2013). Politica, derechos $y$ justicia ambiental. El conflicto del Riachuelo. Buenos Aires: Fondo de Cultura Económica. territarias 40 243 
Ordenanza 44873/91. Argentina. Consejo Deliberante de la Ciudad de Buenos Aires. Desafectación y Afectación de distritos de Zonificación. A varios polígonos de arterias. 12 de marzo de 1991.

Palombi, A. M. (2016). Peregrinos de la Ciudad. Claves para comprender el proceso de relocalización de los habitantes de Villa El Cartón en la Ciudad de Buenos Aires (2005-2014). En Cravino, M. C. (coord.) Detrás de los conflictos. Estudios sobre desigualdad urbana en la Región Metropolitana de Buenos Aires. (pp. 149-178). Los Polvorines: Ediciones Ungs.

Página 12 (2009). Clausura para un cementerio de autos. Diario Página 12. Recuperado de http://www. pagina 12 .com.ar/diario/sociedad/3-125247-2009-05-20.html

Página 12 (30 de julio de 2007). Los más pobres en tierras muy caras. Recuperado de http://www.paginal2.com.ar/ diario/elpais/1-88908-2007-07-30. html
Secretaría de Hábitat e Inclusión de la Ciudad de Buenos Aires (2013). De la villa al barrio. Recuperado de http:// www.buenosaires.gob.ar/habitat/documentos/de-villa-a-barrio

Smulovitz, C. (2008). La política por otros medios. Judicialización y movilización legal en Argentina-. Desarrollo Económico - Revista de Ciencias Sociales, 48(190-191), 287-305.

Theodore, N., Peck, J., \& Brenner, N. (2009). Urbanismo neoliberal: la ciudad y el imperio de los mercados. Temas Sociales, (66), 1-12.

Thomasz, A. (2016). Los nuevos distritos creativos de la ciudad de Buenos Aires: la conversión del barrio de La Boca en el Distrito de las Artes. EURE Revista de Estudios Urbanos y Regionales, 42, 145-167.

Vommaro, G. Morresi, S., \& Belotti, A. (2014). Mundo Pro. Anatomia de un partido fabricado para ganar. Buenos Aires: Planeta. 